\title{
The gap between cell and animal models: nanoparticle drug-delivery development and characterization using microtissue models
}

\author{
"...a nanomaterial and 3D tissue model library becomes important ... \\ in examining both therapeutic and diagnostic agents."
}

Keywords: 3D tissue culture $\|$ drug delivery $\approx$ high throughput $\approx$ multicellular tumor spheroid $\approx$ nanoparticles

Translating therapeutic agents from bench-top research to clinical application undergoes the incremental step from in vitro cell assays to in vivo observations. This large step overlooks many interactions that can be explored in an intermediate model of 3D tissue culture [1]. Improved in vitro models, in the form of $3 \mathrm{D}$ tissue culture, offer a greater insight into biological interactions and lead to improved design of new therapeutics. Many proof-of-principle nanotherapeutic experiments use intratumoral injection of agents to show possible efficacy of treatment. This form of investigation can be assayed in a similar environment to $3 \mathrm{D}$ cultures and has the benefits of avoiding the use of animal subjects. Over the last 60 years, 3D tissue culture and, more specifically, multicellular tumor spheroids have become an important part in understanding cell-cell and cell-matrix interactions. This research has culminated in understanding aspects of tissue formation, phenotypic expression, morphogenic properties, and cellular differentiation. While these 3D tissue properties are being rigorously understood, models that closely resemble in vivo conditions are being developed.

Nanoparticle systems offer multifunctionality for more specialized theranostic techniques [2]. They can be synthesized with controlled intrinsic properties and can be further functionalized with diagnostic, therapeutic or targeting abilities. Their ability to target selected biological characteristics is extremely beneficial for detection [3], greater efficient therapeutic delivery [4] and new routes of delivery [5]. Research in nanomedicine gives the promise of less systemically toxic therapy, early and enhanced detection, and tailored medicine. In this article, we will discuss the benefits and limitations of 3D tissue models for investigating nanoparticle drug delivery.

\section{Current state}

The microenvironment of tissues and cells in diseased states is markedly different than that of traditional in vitro cell culture. Cells adhered to a flat plastic surface enable the study of the direct cellular interaction with nanoparticles, while animal studies offer information on efficacy and toxicity. Neither model explores the interactions with tissue or tissue structures in a controlled manner. Underlying mechanisms of physical interactions with cells, the extracellular matrix (ECM) and concentration gradients are explored in 3D cultures. In addition to the physical interactions, cellular responses and phenotypic expressions change in response to the physical stimuli. For nanoparticle drug delivery, it is important to examine the microenvironment interactions of nanoparticles pertaining to diffusion, uptake and toxicity studies. 3D culture systems that are facile and highly controllable mostly come in the form of cells imbedded in a scaffold matrix [6] or grown in multicellular aggregates $[7,8]$. These systems have merits to explore nanoparticle design and also limitations to mimicking in vivo conditions.

Passive diffusion into 3D structures with cell-cell and cell-matrix interactions varies largely depending on tissue type and the properties of the agent. Initial interaction with the microenvironment depends on ligands or therapeutic agents on the outer surface of the nanoparticle. The $\zeta$-potential and chemical properties of the nanoparticle surface determine the interactions with proteins, ECM and the varying cellular phenotypic expressions, such as receptors and cellular protrusions. In addition to passive diffusive rates, stability of particles can decrease after interactions with ECM and longer transit in interstitial paths, defined as tortuosity. Nanoparticle platforms with

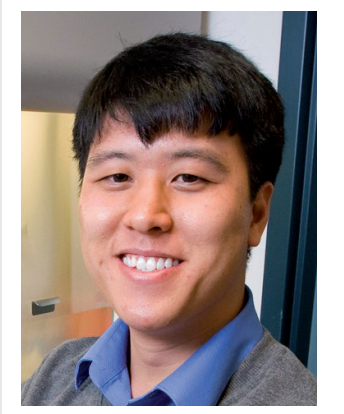

Don N Ho

Department of Chemistry, Brown

University, Providence, RI, USA

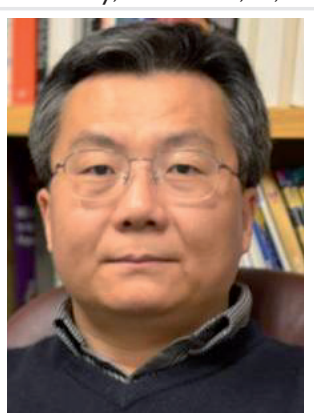

Shouheng Sun

Author for correspondence: Department of Chemistry, Brown University, Providence, RI, USA

E-mail: ssun@brown.edu 
sizes ranging from molecular to viral are in the regime of 1-100 nm, and this size variation also creates differences in diffusion and surface contact dynamics. Scaffold matrix culture systems examine interactions with nanoparticles using controlled ratios of ECM, natural products, and biodegradable or degradable components with embedded cells. Multicellular aggregate-based systems can interrogate native ECM production and cellular interaction. For nanoparticle design, varying ligands or externally functionalized drugs alter passive kinetics and can be directly quantified. Diffusion of nanoparticles has been observed to be modulated by size, surface functionalization of collagenase on the surface of nanoparticles and collagenase treatment of multicellular tumor spheroids [9]. Measurement of diffusive ability can be accomplished by fixation and sectioning to measure penetration distance. Diffusion rates can be directly measured with fluorescent recovery after photobleaching and confocal microscopy [10] . Diffusion characteristics are the dominant factors in nanoparticle designs that rely on increased local concentrations due to the enhanced permeability and retention (EPR) effect [11,12]. The characterization of passive diffusion helps to understand basic microenvironment interactions and further optimize nanoparticle delivery.

Targeting ability is altered by phenotypic expression, 3D diffusion and matrix interaction, much like the diffusion processes. Greater resolution to specificity, cell interaction and ECM interaction can be examined in 3D tissue culture. With the customizable nanoscale delivery platforms, these molecule-sized agents can obtain both targeted diagnostics and targeted therapy. The addition of specific targeting agents alters the penetration depth and accumulation of nanoparticles. Targeting tumor vasculature has shown promise to inhibit the transport of oxygen and nutrients to cancerous cells as a mode of treatment. Endothelial cell-targeted ironoxide nanoparticles have been found to associate specifically with newly formed endothelial cell coating on a multicellular tumor spheroid [7]. Mechanistic studies can optimize targeting and find underlying interactions that can maximize delivery of nanoparticles to desired targets. Targeting design and also further innovation into areas such as multi-stage delivery can benefit from understanding microenvironment interactions in 3D tissue culture.

Toxicity and therapeutic capacity of agents changes from traditional $2 \mathrm{D}$ cell culture and $3 \mathrm{D}$ tissue culture due to the diffusion gradients and phenotypic expression in tissue. For example, hepatocytes have reduced functional ability to metabolize products and produce proteins in $2 \mathrm{D}$ cell culture, but regain this trait when grown in 3D [13]. Similarly, toxicity of agents can be altered by both phenotypic expression and diffusion of agents; an agent could be more or less toxic when changing in vitro and in vivo models. Cellular uptake is also altered as cell physical dimensions (i.e., cell-cell and cell-ECM contact)and phenotypic pathways for gene expression, uptake, metabolism and proliferation are modified [14]. Toxicity measurements in 3D tissue cultures have been found to more closely resemble results found with in vivo models [15].

\section{"Theranostic application of nanoparticles uses the multifunctional ability to both deliver therapy and track progress of disease..."}

Theranostic application of nanoparticles uses the multifunctional ability to both deliver therapy and track progress of disease, allowing for interrogating and tailoring to certain disease states and individual patients [4]. Development of diagnostic-agent lifetime, in addition to efficacy of the therapeutic agent, can be monitored in $3 \mathrm{D}$ tissue culture. The degradation of the nanoparticle system, stability of diagnostics modes, and tracking of therapeutic location and efficiency can be defined in the microenvironment.

\section{Advantages \& limitations}

Cost and ethical benefits of 3D tissue culture are from a reduced number of animal subjects. Greater mechanistic understanding of an agent's biological interactions improves design and provides greater insight going into in vivo studies. The economic cost to obtain and prepare animal models, and to have devoted animal care facilities, is an initial incentive to optimize nanoparticle therapy design before going into extensive studies that usually show markedly reduced effects. In addition, the ethical responsibility to minimize the use of animals in research makes optimizing therapeutic design a necessity.

3D tissue culture can replicate specific microenvironments and elucidate these interactions. Certain humoral responses can be introduced to incorporate some systemic responses, but it must be noted that some interactions can only be explored in an animal model. Protein and molecule interactions on the surface of nanoparticles 
affect biological interactions. The surface of synthesized particles can be altered in vivo so that proteins coat the outer surface and this interaction must also be properly examined [16]. Applying appropriate proteins to cell culture media can mimic these interactions, but care must be taken as to not affect the 3D tissue model. Perfusion, flow and pressure are largely static for in vitro culture models. Beyond basic diffusion, EPR models, flow and pressure are implemented with bioreactors and, more recently, microfluidic systems. These systems have been used to examine changes in flow, mimic interstitial pressure and introduce environments such as those present in EPR that are found in vivo and affect delivery of agents [6]. While for complex cell-cell interactions, cocultures can be used in 3D tissue culture to study controlled cellular interactions. The addition of other supporting cell types, such as fibroblasts, endothelial cells and astrocytes, gives greater similarity to the heterogeneous composition found in living tissues by adding both cellular interactions and signaling cues. However, co-cultures can become difficult to control and mimicking complex biological systems requires greater time for the development of these heterogeneous models. Ex vivo models offer examination of $3 \mathrm{D}$ tissue that is the exact biological environment of interest. Although these models still require animals, the intermediate phase of closely matching $3 \mathrm{D}$ tissue

\section{References}

1 Kunz-Schughart LA. Multicellular tumor spheroids: intermediates between monolayer culture and in vivo tumor. Cell Biol. Int. 23(3), 157-161 (1999).

2 Ho D, Sun X, Sun S. Monodisperse magnetic nanoparticles for theranostic applications. Acc. Chem. Res. 44(10), 875-882 (2011).

$3 \mathrm{Xu}$ C, Xie J, Ho D et al. Au-Fe $\mathrm{O}_{4}$ dumbbell nanoparticles as dual-functional probes. Angew Chem. Int. Ed. Engl. 47(1), 173-176 (2008).

4 Xu C, Wang B, Sun S. Dumbbell-like $\mathrm{Au}-\mathrm{Fe} 3 \mathrm{O} 4$ nanoparticles for target-specific platin delivery. J. Am. Chem. Soc. 131(12), 4216-4217 (2009).

5 Xu C, Yuan Z, Kohler N, Kim J, Chung MA, Sun S. FePt nanoparticles as an Fe reservoir for controlled Fe release and tumor inhibition. J. Am. Chem. Soc. 131(42), 15346-15351 (2009).

$6 \quad \mathrm{Ng}$ CP, Pun SH. A perfusable 3D cell-matrix tissue culture chamber for in situ evaluation of nanoparticle vehicle penetration and transport. Biotechnol. Bioengineering 99(6), 1490-1501 (2008).

culture systems to in vivo models would benefit from correlating results.

\section{Conclusion}

Once nanoplatforms have been well characterized in 3D tissue culture, combinations from optimal designs can be quickly implemented in brief $3 \mathrm{D}$ assays before continuing to an animal model. The idea of producing a nanomaterial and 3D tissue model library becomes important for sound scientific practice and efficient research [17]. Similar to the bank of animal models and high-throughput small-molecule discovery, resources for an array of $3 \mathrm{D}$ in vitro studies can be established for certain diseases in the context on nanoparticle drug delivery. These models can become important in examining both therapeutic and diagnostic agents.

\section{Financial \& competing interests disclosure}

This work was supported in part by the US Department of Education through GAANN Award P200A090076, administered by the Institute for Molecular and Nanoscale Innovation at Brown University. The authors have no other relevant affiliations or financial involvement with any organization or entity with a financial interest in or financial conflict with the subject matter or materials discussed in the manuscript apart from those disclosed.

No writing assistance was utilized in the production of this manuscript.

7 Ho DN, Kohler N, Sigdel A et al. Penetration of endothelial cell coated multicellular tumor spheroids by iron oxide nanoparticles. Theranostics 2(1), 66-75 (2012).

8 Napolitano AP, Dean DM, Man AJ et al. Scaffold-free three-dimensional cell culture utilizing micromolded nonadhesive hydrogels. Biotechniques 43(4), 494, 496-500 (2007).

9 Goodman TT, Olive PL, Pun SH. Increased nanoparticle penetration in collagenasetreated multicellular spheroids. Int. J. Nanomed. 2(2), 265-274 (2007).

10 Berk DA, Yuan F, Leunig M, Jain RK. Fluorescence photobleaching with spatial Fourier analysis: measurement of diffusion in light-scattering media. Biophysi. J. 65(6), 2428-2436 (1993).

11 Iyer AK, Khaled G, Fang J, Maeda H. Exploiting the enhanced permeability and retention effect for tumor targeting. Drug Discov. Today 11(17-18), 812-818 (2006).

12 Brannon-Peppas L, Blanchette JO. Nanoparticle and targeted systems for cancer therapy. Adv. Drug Deliv. Rev. 56(11), 1649-1659 (2004).
13 Hamilton G, Westmoreland C, George E. Effects of medium composition on the morphology and function of rat hepatocytes cultured as spheroids and monolayers. In Vitro Cell. Dev. Biol. Anim. 37(10), 656-667 (2001).

14 Kim J, Stein R, O’Hare M. Threedimensional in vitro tissue culture models of breast cancer - a review. Breast Cancer Res. Treat. 85(3), 281-291 (2004).

15 Lee J, Lilly GD, Doty RC, Podsiadlo P, Kotov NA. In vitro toxicity testing of nanoparticles in 3D cell culture. Small 5(10), 1213-1221 (2009).

16 Walkey CD, Chan WCW. Understanding and controlling the interaction of nanomaterials with proteins in a physiological environment. Chem. Soc. Rev. 41(7), 2780-2799 (2012).

17 Weissleder R, Kelly K, Sun EY, Shtatland T, Josephson L. Cell-specific targeting of nanoparticles by multivalent attachment of small molecules. Nat. Biotechnol. 23(11), 1418-1423 (2005). 